\title{
Assembly and Activation of the MotA/B Proton Channel Complex of the Proton-Driven Flagellar Motor of Salmonella enterica
}

\author{
Yusuke V. Morimoto and Tohru Minamino \\ Osaka University \\ Japan
}

\section{Introduction}

Salmonella enterica can swim by rotating multiple flagella, which arise randomly over the cell surface (Fig. 1A). The flagellum consists of at least three parts: the basal body, the hook, and the filament. The basal body is embedded within the cell membranes and acts as a bidirectional rotary motor powered by an electrochemical potential gradient of protons across the cytoplasmic membrane (Fig. 1B, C). The hook and filament extend outwards in the cell exterior. The filament acts as a helical propeller. The hook exists between the basal body and filament and functions as a universal joint to smoothly transmit torque produced by the motor to the filament. When the motors rotate in counterclockwise direction, the cells can swim smoothly. By quick reversal rotation of the motor to clockwise direction, the cells tumble and change their swimming direction to move toward more favorable environments (Fig. 2) (Berg, 2003; Blair, 2003; Minamino et al., 2008).

In Salmonella enterica, five flagellar proteins, MotA, MotB, FliG, FliM and FliN, are responsible for torque generation. Two integral membrane proteins, MotA and MotB, form the stator complex of the motor, which functions as a proton-conducting channel to convert an inwardly directed flux of protons through the proton channel into the mechanical work required for motor rotation. Two highly conserved residues, Pro-173 in MotA and Asp-33 in MotB, are involved in the energy coupling mechanism. FliG, FliM and FliN form the C-ring on the cytoplasmic face of the MS ring and are responsible not only for torque generation but also for switching the direction of motor rotation (Berg, 2003; Blair, 2003; Minamino et al., 2008). Torque is generated by sequential electrostatic interactions between MotA and FliG. A high-resolution observation of flagellar motor rotation has revealed a fine stepping motion with 26 steps per revolution, which corresponds to that of FliG subunits in the Cring (Sowa et al., 2005; Nakamura et al., 2010). The proton conductivity of the MotA/B proton channel complex is proposed to be suppressed by a plug segment of MotB when the MotA/B complex is not assembled around the basal body (Hosking et al., 2006; Morimoto et al., 2010a). Although a low-resolution structure of the entire basal body containing the stator complexes has been visualized in situ by electron cryotomography (Fig. 3A) (Murphy et al., 2006; Liu et al., 2009; Kudryashev et al., 2010), the stators are missing in highly purified flagellar basal bodies presumably due to highly dynamic interactions between the stator and its binding partners in the basal body (Fig. 3B) (Thomas et al., 2001, 2006). In this chapter, we describe our current understanding of how the stator complex is installed into the motor, 
how its proton conductivity is activated, and how the proton flow through the proton channel is coupled with torque generation.

A

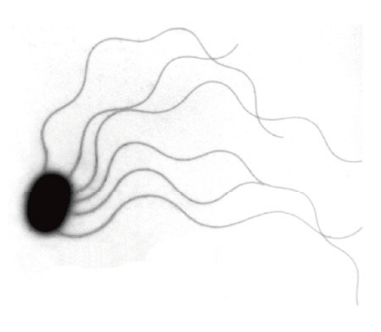

B

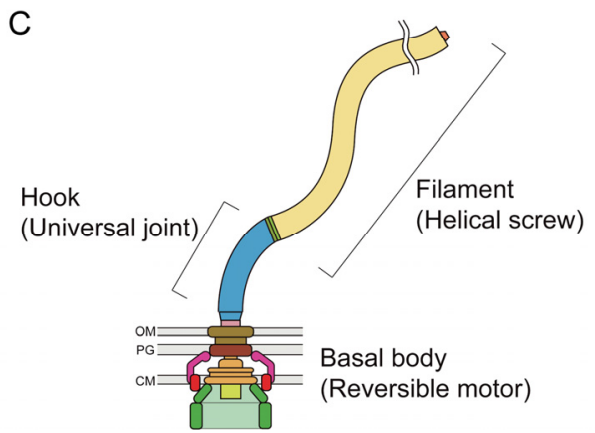

Fig. 1. Bacterial flagella

(A) Electron micrograph of Salmonella enterica. Six flagella arise randomly over the cell surface.

(B) Electron micrograph of the purified flagellum. (C) Schematic diagrams of the flagellum.

The flagellum consists of at least three parts: the basal body, which acts as a reversible rotary motor; the hook, which functions as a universal joint; and the filament, which acts as a helical screw. OM, outer membrane; PG, peptidoglycan layer; CM, cytoplasmic membrane.

Typical swimming pattern of Salmonella cells. The cells can swim smoothly when the motors rotate in counterclockwise (CCW) direction, resulting in the formation of a flagellar bundle that produces the thrust. By quick reversal rotation of the motor to clockwise $(\mathrm{CW})$ direction, the bundle is disrupted so that bacteria can tumble and change their swimming direction.

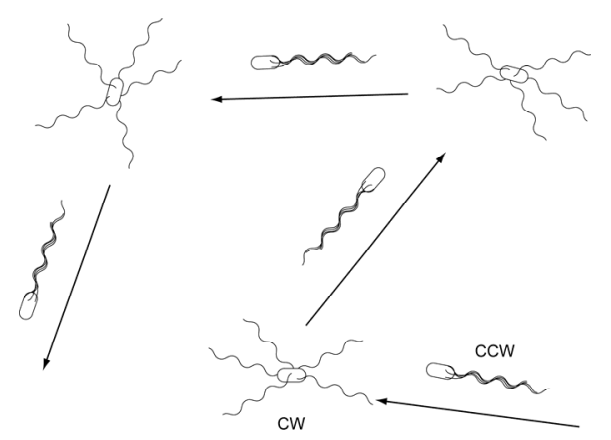

Fig. 2. Bacterial behavior 


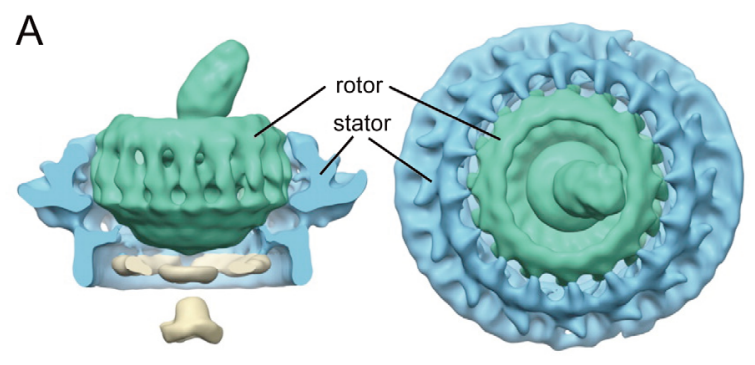

B
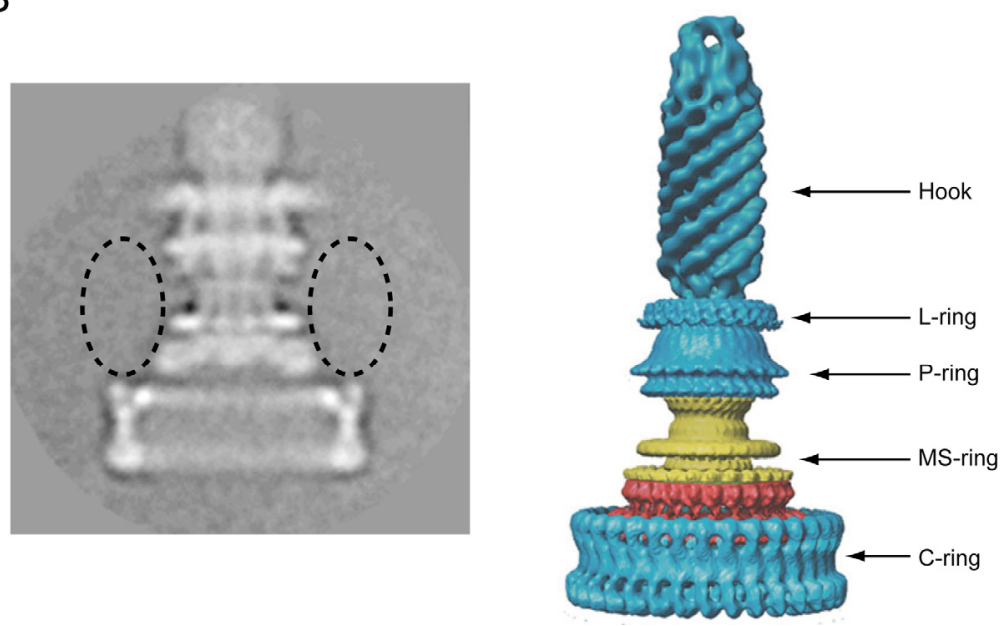

Fig. 3. Structure of bacterial flagellar motor

(A) The entire structure of the Borrelia burgdorferi flagellar motor visualized by electron cryotomography. The segmented structure was color coded as follows: rotor, green; C-ring and stator, blue; protein export apparatus, gray (Liu et al., 2009). (B) Three-dimensional (3D) structure of the flagellar basal body isolated from Salmonella enterica (Thomas et al., 2006). Left panel: electron cryomicroscopic image of frozen-hydrated hook-basal body in the side view. Right panel: 3D density map of the basal body reconstructed from frozen-hydrated particle images. Dashed circles indicate putative positions of the stator complexes in the motor.

\section{MotA/B stator complex acts as a transmembrane proton channel}

MotA and MotB are cytoplasmic membrane proteins and form a complex consisting of four copies of MotA and two copies of MotB (Kojima and Blair, 2004). It has been estimated that there are at least 11 copies of the MotA/B complex around the flagellar basal body (Reid et al., 2006). Since MotB has a potential peptidoglycan-binding motif in its C-terminal periplasmic domain (De Mot and Vanderleyden, 1994), the MotA/B complex is postulated to be anchored to the peptidoglycan (PG) layer to act as the stator of the motor. The MotA/B complex forms a transmembrane proton channel that couples an inward-directed proton translocation to torque generation (Blair and Berg, 1990). However, the molecular mechanism of energy coupling between proton influx and flagellar motor rotation remains unknown. 
The torque-speed relationship of the flagellar motor has been well characterized (Fig. 4)(Chen and Berg, 2000a, b). As the load is decreased, torque decreases gradually up to a certain speed called the knee point and then falls rapidly to zero. The rotation rate of the proton-driven flagellar motor under a given load is proportional to a proton motive force across the cytoplasmic membrane over the entire range of observation (Gabel and Berg, 2003). The plateau torque at high load is dependent on the number of stators while the motor speed near zero load is independent of the stator number (Fig. 4A) (Ryu et al., 2000; Yuan and Berg, 2008). In the high-torque, low-speed regime, both temperature and solvent-isotope effects are small, while those effects are large in the low-torque, highspeed regime. This suggests that the steep decline of torque at high speed is due to a limit in the rate of proton transfer (Fig. 4B) (Chen and Berg, 2000a, b).
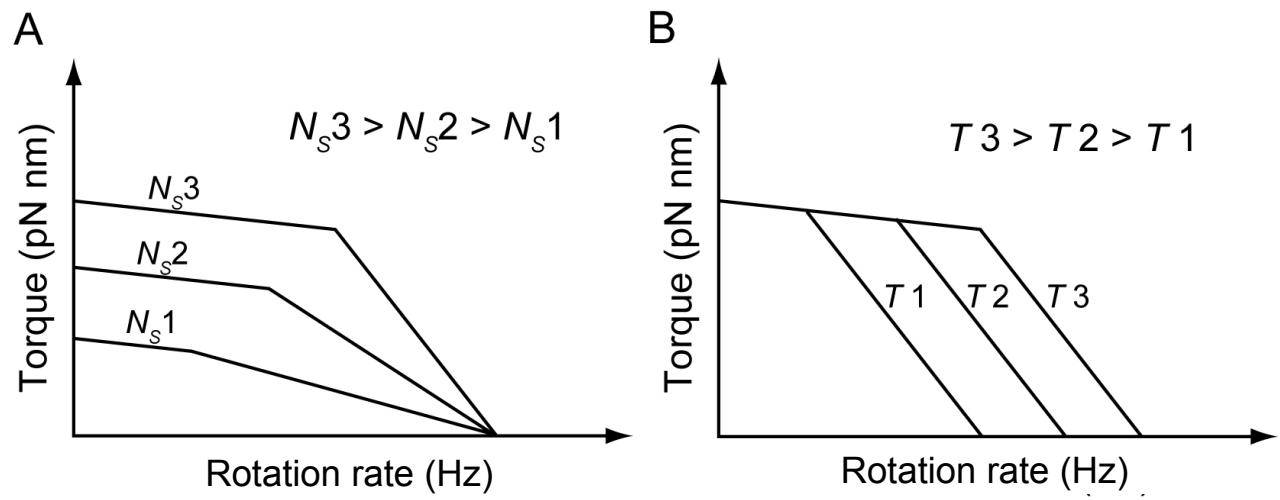

Fig. 4. Torque-speed curve of the bacterial flagellar motor

(A) Effect of the number of functional stators in a motor on the torque-speed relationship. The torque at high load is dependent on the number of stators. The motor speed near zero load is independent of the functional stator number. (Ryu et al., 2000) (B) Effect of temperature on the torque-speed curve. In the low-speed regime, torque is insensitive to changes in temperature. In the high-speed regime, motor speed decreases markedly when temperature is decreased (Chen et al., 2000a).

\subsection{Arrangement of transmembrane segments of the MotA/B complex}

MotA consists of four transmembrane spans (TMs), two short periplasmic loops and two extensive cytoplasmic regions. MotB consists of an N-terminal cytoplasmic region, one TM and a large periplasmic region containing a putative peptidoglycan-binding motif (Fig. 5A). The four MotA subunits in the stator complex are positioned with their TM3 and TM4 segments adjacent to the dimer of MotB-TM located at the center and their TM1 and TM2 segments in outer positions (Fig. 5B) (Braun et al., 2004). Two conserved charged residues, Arg-90 and Glu-98, in the cytoplasmic loop between TM2 and TM3 are involved in electrostatic interactions with charged residues in the C-terminal domain of FliG to produce torque (Zhou and Blair, 1997; Zhou et al., 1998a). A conserved proline residue of MotA, Pro173 and Pro-222, are thought to be involved in conformational changes of the stator complex that couple proton influx with torque generation (Braun et al., 1999; Nakamura et al., 2009b). The absolutely conserved and functionally critical aspartic acid residue, Asp-33 of MotB is 
located near the cytoplasmic end of its TM and is postulated to be a proton-binding site (Sharp et al., 1995; Togashi et al., 1997; Zhou et al., 1998b; Che et al., 2008; Morimoto et al., 2010a). MotB exists as a dimer in the stator complex and these aspartic acid residues are positioned on the surface of the MotB-TM dimer facing MotA-TMs, suggesting that the stator complex is likely to have two proton-conducting pathways (Fig. 5B) (Braun and Blair, 2001). Both protonation and deprotonation of this aspartic acid residue cause conformational changes of the cytoplasmic loop of MotA, which may drive flagellar motor rotation (Kojima and Blair, 2001; Che et al., 2008).
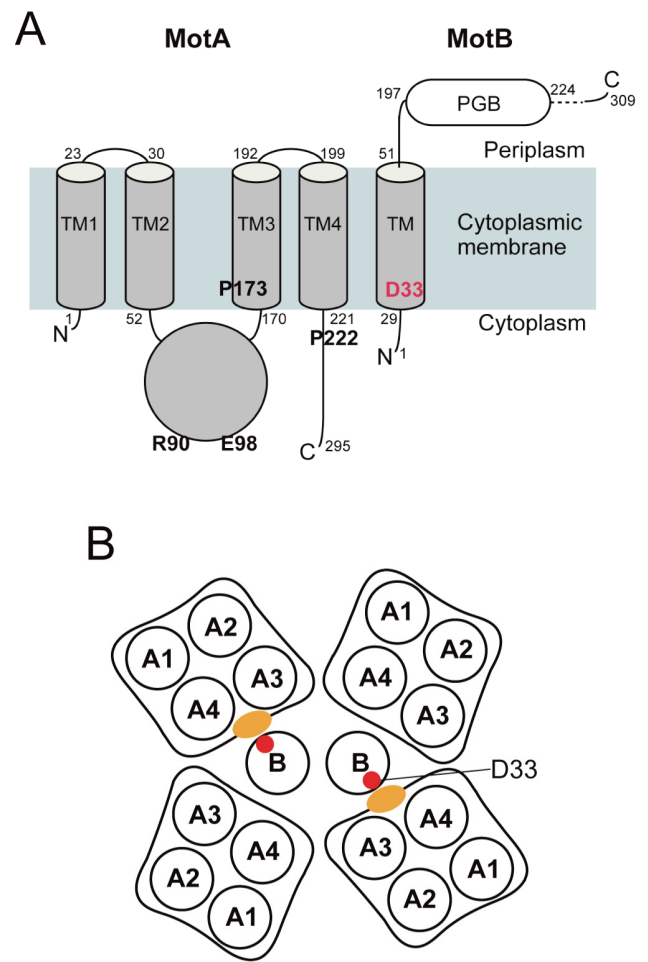

Fig. 5. Topology of MotA and MotB

(A) Cartoon representing the domain organization of MotA and MotB. Salmonella MotA and MotB consist of 295 and 309 amino acid residues, respectively. MotA has four transmembrane segments (TM1-TM4), two short periplasmic loops and two large cytoplasmic regions. MotB has an N-terminal cytoplasmic region, one transmembrane segment and a large periplasmic domain containing a putative peptidoglycan-binding motif (PGB) (Braun et al., 1999, 2004; Kojima et al., 2004). Arg-90 and Glu-98 of MotA are required for electrostatic interactions with FliG. Pro-173 and Pro-222 of MotA are proposed to be responsible for conformational changes of the stator complex that couple proton influx with torque generation. Asp-33 in MotB is postulated to play an important role in proton translocation. (B) Arrangement of transmembrane segments of the MotA/B complex, which consists of 4 copies of MotA and 2 copies of MotB. The view is from the periplasmic side of the membrane. The complex has two proton conducting pathway shown by orange ellipsoids. (Braun et al., 2004). 


\subsection{Asp-33 of MotB and Pro-173 of MotA are responsible for energy coupling between proton flow and motor rotation}

Asp-33 of MotB is a highly conserved and protonatable residue, and is believed to be involved in the proton-relay mechanism (Sharp et al., 1995; Togashi et al., 1997; Zhou et al., 1998b). An increase in the intracellular proton concentration suppresses or even abolishes the flagellar motor function. This suggests that a decrease in the intracellular $\mathrm{pH}$ presumably interferes with proton dissociation from Asp-33 to the cytoplasm, thereby slowing the torque generation reaction cycle of the motor (Minamino et al., 2003; Nakamura et al., 2009a). Therefore, it seems likely that both protonation and deprotonation of this Asp play key roles in the torque generation cycle. A D33E mutation in MotB causes ca. $40 \%$ reduction in stall torque and a sharp decline in the torque-speed curve with an apparent maximal rotation rate of ca. $20 \mathrm{~Hz}$. This suggests that the D33E mutation not only reduces the proton conductivity significantly but also interferes with an actual torque generation step by the stator-rotor interactions considerably (Che et al., 2008). The stall torque is recovered nearly to the wild-type levels by the suppressor mutations in the transmembrane helices TM2 and TM3 of MotA and in TM and the periplasmic domain of MotB. In contrast, high-speed rotation under low load is still significantly limited even in the presence of these suppressor mutations. These suggest that the second-site mutations recover energy coupling of an inward-directed proton translocation with torque generation but not the maximum proton conductivity (Che et al., 2008).

Pro-173 is highly conserved among MotA orthologs. Since point mutations at Pro-173 affect not only motor function but also proton flow through the MotA/B complex, this prolyl residue seems to play a critical role in the proton-relay mechanism (Braun et al., 2004). E. coli $\operatorname{mot} A(\mathrm{P} 173 \mathrm{C}) / \operatorname{motB}(\mathrm{D} 32 \mathrm{C})$ double mutant strain gives a high yield of disulfide-linked MotA/B heterodimers upon oxidation with iodine. This suggests that Pro-173 of MotA is positioned in the proton channel near Asp-32 of MotB, which corresponds to Asp-33 in Salmonella MotB (Braun et al., 2004). The Salmonella motA(P173A) mutant produces stall torque at wild-type levels (Nakamura et al., 2009b). However, the P173A mutation causes a sharp decline in the torque-speed curve with a maximum rotation rate of ca. $25 \mathrm{~Hz}$ (Nakamura et al., 2009b). These suggest that the P173A mutation reduces the maximum proton conductivity of the MotA/B complex but not the efficiency of energy coupling between proton translocation and torque generation. Since Pro-173 of MotA is likely to be very close to Asp-33 of MotB in the MotA/B complex structure (Braun et al., 2004), and because Pro is known to induce a kink in $\alpha$-helix, its replacement with Ala would relax the conformational strain of the MotA/B complex, resulting in a change in protein dynamics to slow down the rates of conformational changes that switch the exposure of Asp-33 to the outside or the inside of the cell. Therefore, Pro- 173 is proposed to play an important role in facilitating the resetting of the position of Asp-33 relative to the proton pathway in the conformational change between the two distinct states to facilitate proton translocation when the motor spins at high speed (Nakamura et al., 2009b).

\subsection{Role of conserved charged residues in MotA in stator assembly around the motor}

Over-expression of MotA inhibits motility of wild-type cells. Neither cell growth, flagellar formation, nor proton motive force across the cytoplasmic membrane is affected by overproduced MotA. However, stall torque is significantly reduced. Since the stall torque is 
dependent on the number of functional stators in the motor (Fig. 4), this suggests that MotA occupies the stator-binding sites of the motor and reduces the number of functional stators (Morimoto et al., 2010b). In agreement with this, fluorescent spots of MotA-mCherry have been observed at the same position as those of GFP-FliG even in the absence of MotB (Fig. 6) (Morimoto et al., 2010b). Thus, MotA alone can be installed into a motor.

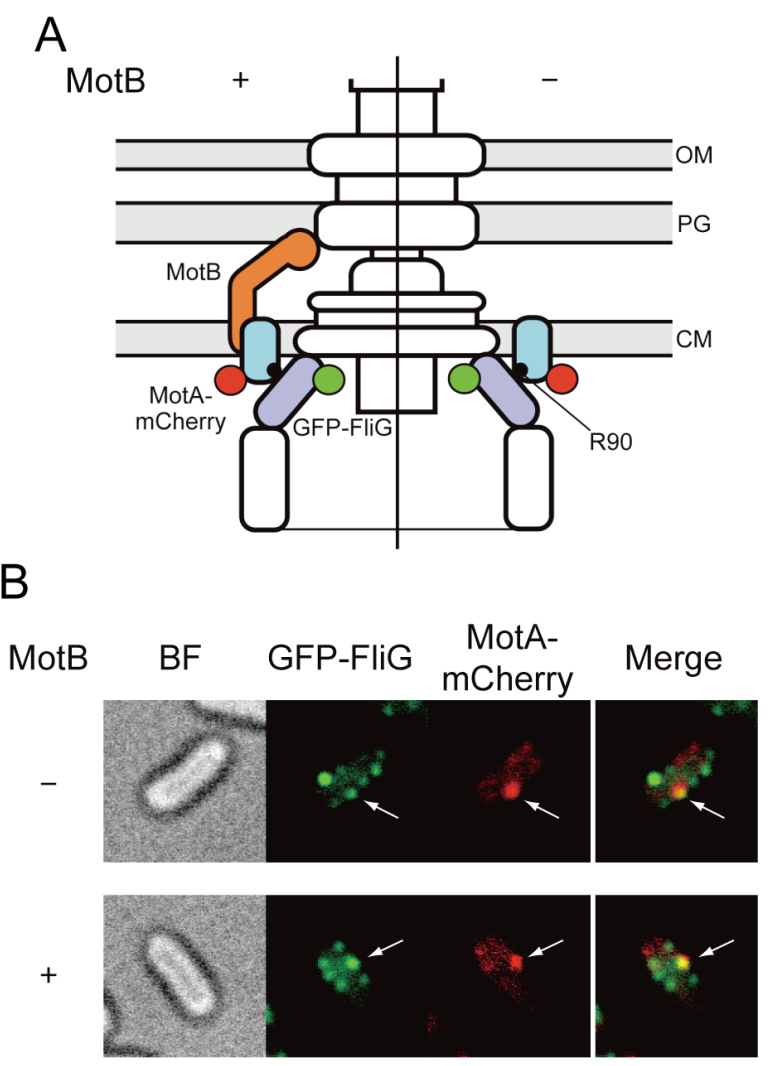

Fig. 6. Co-localization of GFP-FliG and MotA-mCherry

(A) Cartoon representing the basal body and positions of GFP-FliG and MotA-mCherry in the presence $(+)$ or absence $(-)$ of MotB. (B) Bright-field (BF) and fluorescence images of GFP-FliG and MotA-mCherry observed by fluorescence microscopy. The fluorescence images of GFPFliG (green) and MotA-mCherry (red) are merged in the right panel. (Morimoto et al., 2010b).

Two charged residues of MotA, Arg-90 and Glu-98, are involved in electrostatic interactions with charged residues of FliG for torque generation (Zhou \& Blair, 1997; Zhou et al., 1998a). The $\operatorname{mot} A(\mathrm{R} 90 \mathrm{E})$ and $\operatorname{mot} A(\mathrm{E} 98 \mathrm{~K})$ alleles are recessive and hence do not exert a negative dominance when their expression levels were the same as those of wild-type cells (Zhou \& Blair, 1997; Morimoto et al., 2010b). Interestingly, however, an increase in the expression level of the MotA(R90E)/B complex by more than 10-fold allows $70 \%$ of the cells to swim in liquid media (Morimoto et al., 2010b). In agreement with this, the R90E mutation markedly decreases the number and intensity of fluorescent spots of GFP-MotB. Since the loss-of- 
function phenotype of the $\operatorname{mot} A(\mathrm{R} 90 \mathrm{E})$ and $\operatorname{mot} A(\mathrm{E} 98 \mathrm{~K})$ alleles are considerably suppressed by the $f l i G(\mathrm{D} 289 \mathrm{~K})$ and $f l i G(\mathrm{R} 281 \mathrm{~V})$ mutations, respectively (Zhou et al., 1998a), this suggests that the interactions between MotA Arg-90 and FliG Asp-289 and between MotA Glu-98 and FliG Arg-281 are critical not only for torque generation but also for the assembly of the stators into the motor (Morimoto et al., 2010b).

Polar localization of the PomA/B complex of Vibrio alginolyticus, which are homologs of the MotA/B complex and acts as the stator of the motor fueled by the sodium motive force across the cytoplasmic membrane, is greatly affected by changes in the external concentration of sodium ions (Fukuoka et al., 2009). This suggests that sodium ions are required not only for torque generation but also for the efficient assembly process of PomA/B. In contrast, neither the D33N nor D33A mutation in Salmonella MotB, which abolishes the proton flow through a proton channel, affects stator assembly around the motor, indicating that stator assembly in the proton-driven motor is not dependent on the proton conductivity of the MotA/B complex. In addition, depletion of the proton motive force across the cytoplasmic membrane by a protonophore, carbonyl cyanide $m$ chlorophenylhydrazone does not abolish the subcellular localization of the stator labeled with GFP, indicating that the stators remain to exist around the rotor even in the absence of the proton motive force. This is supported by the finding that the stator actually switches its functional state between the active and inactive ones without detaching from the rotor completely when PMF is largely reduced (Nakamura et al., 2010). Therefore, it is much likely that the assembly of the Salmonella MotA/B complex into the motor is not obligatorily linked to the process of the proton translocation through the proton channel of the MotA/B complex.

\subsection{MotB $\mathrm{B}_{\mathrm{C}}$ controls the proton channel activity during stator assembly}

The periplasmic domain of MotB $\left(\operatorname{Mot}_{\mathrm{C}}\right)$ consisting of residues 78 through 309 Kojima et al., 2008 contains a putative peptidoglycan-binding (PGB) motif, which shows sequence similarity to other Omp-like proteins (Fig. 7A) (De Mot and Vanderleyden, 1994). Interestingly, the crystal structure of the core domain of Salmonella MotB $\mathrm{C}_{\mathrm{C}}$ which consists of residues 99 through 276 has a typical OmpA-like structure, and shows considerable structural similarities to the C-terminal regions of PAL and RmpM (Fig. 7A) (Kojima et al., 2009). Mutations in the PGB motif of MotB significantly impair motility, indicating that anchoring MotA/B complex to the PG layer is critical for the motor function. Stator resurrection experiments have shown that abrupt drops in the rotation rate occur frequently even in steadily rotating motors (Block and Berg, 1984; Blair and Berg, 1988; Sowa et al., 2005). Consistently, fluorescent photo-bleaching studies of GFP-fused MotB have shown a rapid exchange and turnover of the stator complexes between the membrane pool and the basal body (Leake et al., 2006). These results suggest that the association between MotA/B and its target site on the basal body are highly dynamic.

MotB $_{C}$ forms homo-dimer in solution (Fig. 7B) (Kojima et al., 2008, 2009). Over-expression of Mot $B_{C}$ inihibits motility of wild-type cells when expressed in the periplasm. An in-frame deletion of resiudes 197-210 not only inhibits dimerization of $\operatorname{Mot}_{C}$ but also significantly reduces its inhibitory effect on wild-type motility. These results suggest that dimerization of Mot $\mathrm{B}_{\mathrm{C}}$ is responsible for the proper targeting and stable anchoring of the MotA/B complex to the putative stator binding sites of the basal body (Kojima et al., 2008). Recently, sitedirected disulfide crosslinking experiments have shown an interaction between $\operatorname{Mot}_{C}$ and the P ring of the basal body (Hizukuri et al., 2010). 

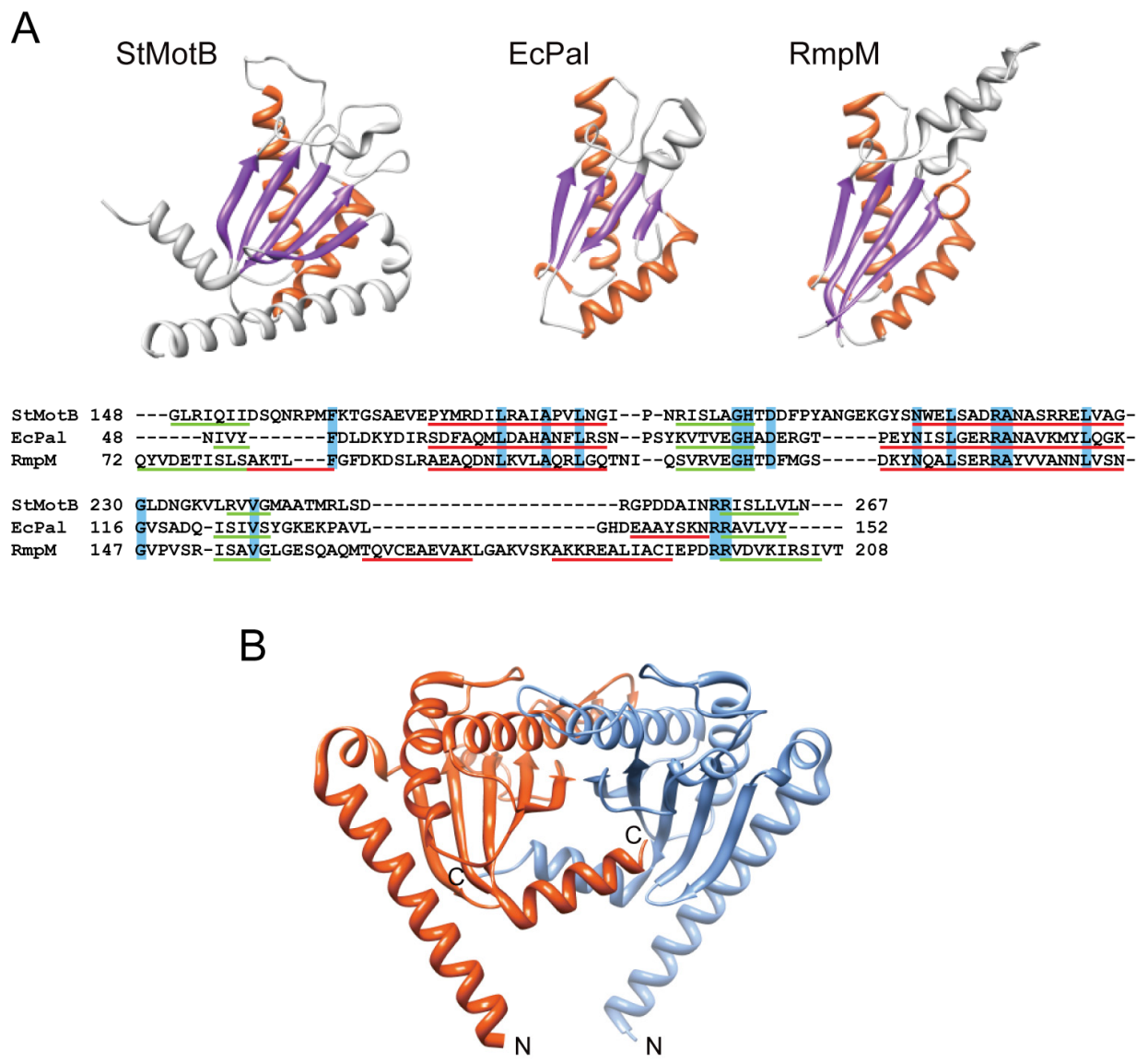

Fig. 7. Crystal structure of the C-terminal domain of MotB

(A) Comparison of various OmpA-like domains. Ca ribbon diagrams of three OmpA-like domains and their structure-based sequence alignment. StMotB, Salmonella typhimurium $\operatorname{MotB}_{\mathrm{C} 2}$ (2zvy); EcPal, Escherichia coli Pal (1oap); RmpM, the C-terminal domain of RmpM from Neisseria meningitidis (1r1m). Conserved secondary structural elements are colored: orange, a-helix; purple, $\beta$-strand. Residues highlighted in light blue in the aligned sequences are conserved in these three proteins. Regions of secondary structures are indicated below the corresponding sequences: red line, a-helix; green line, $\beta$-strand. (B) Ca ribbon representations of the $\operatorname{MotB}_{\mathrm{C} 2}$ dimer. The two subunits are shown in blue and orange. (Kojima et al., 2009)

Cell growth is not impaired significantly by over-expression of the E. coli MotA/B complex (Stolz and Berg, 1994). In contrast, the cell growth is severely impaired by co-expression of MotA with a MotB-TetA chimera protein, in which the first 60 residues of MotB are fused to a 50 residue sequence encoded by an open reading frame in the tet $A$ gene (Blair and Berg, 1990). Since the replacement of Asp-32 to other residues in the MotB-TetA chimera protein has been shown to suppress proton leakage caused by co-overproduction of MotA with the 
MotB-TetA chimera protein. Therefore, it has been suggested that the proton conductivity of the MotA/B complex is suppressed prior to stator assembly around a motor. An in-frame deletion of residues 51-70 or 52-71 within the periplasmic domain of E. coli MotB or Salmonella MotB, respectively, a region, which is highly conserved among the MotB orthologs, causes considerable proton leakage, thereby arresting cell growth (Hosking et al., 2006; Morimoto et al., 2010a). This suggests that the deleted region of MotB acts as a plug segment that inserts into a proton channel within the cytoplasmic membrane to prevent from premature proton translocation through the channel before the association with a motor and that upon stator assembly in the motor, the plug leaves the channel, allowing the stator to conduct protons (Fig. 8). Interestingly, however, cell growth is not significantly impaired by in-frame deletion of residues 51-100 in Salmonella MotB, which contains the plug segment (Muramoto and Macnab, 1998; Kojima et al., 2009). This indicates that a proton channel of the MotA/B $(\Delta 51-100)$ complex is not formed prior to its assembly around the rotor although the plug is missing. The introduction of the L119P or L119E substitution into $\operatorname{MotB}(\Delta 51-100)$ causes growth impairment due to significant proton leakage (Kojima et al., 2009; Morimoto et al., 2010a). Therefore, it is likely that some other region within the periplasmic domain of MotB also regulates proper formation of the proton channel during stator assembly.

\section{open closed}

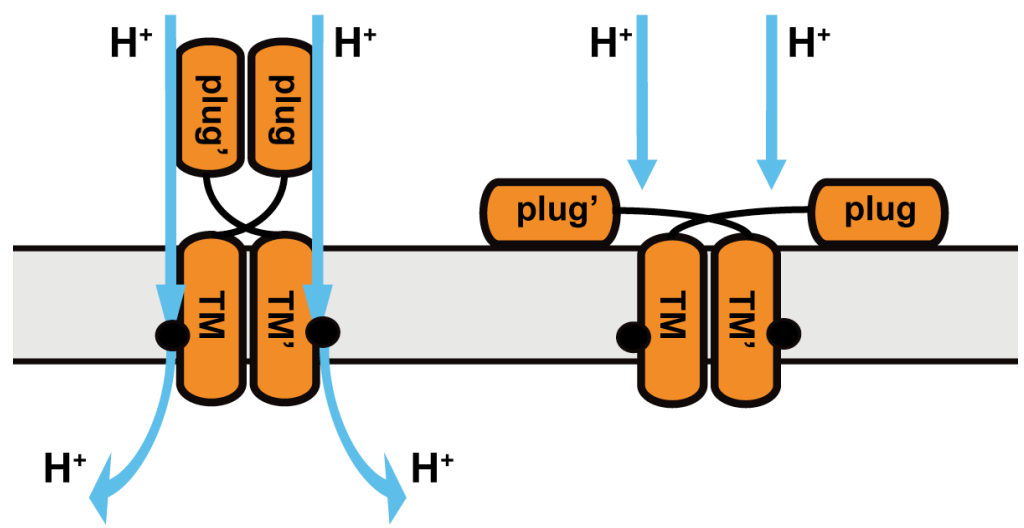

Fig. 8. Schematic diagram of the plug region of MotB

Cartoon representing the position of the plug in the open and closed states of the MotA/B proton channel. Only the MotB TMs and plugs are shown, in a view parallel with the plane of the cytoplasmic membrane. Asp-33 in MotB is shown as a black dot. Blue arrows indicate the proton flow through proton channels. In the open state, the plugs leave the membrane and associate with each other via their hydrophobic faces to hold the proton channel open. In the closed state, the plugs insert into the cell membrane parallel with its periplasmic face and interfere with channel formation (Hosking et al., 2006).

$\operatorname{MotB}(\Delta 51-100)$ is still functional, indicating that $\operatorname{MotB}(\Delta 51-100)$ can form a functional stator complex along with MotA (Muramoto and Macnab, 1998). Since the distance between the 
surface of the hydrophobic core layer of the cytoplasmic membrane and that of the PG layer is about $100 \AA$, and the crystal structure of the core domain of MotB $B_{C}$ is only about $50 \AA$ tall, the crystal structure of the core domain of Salmonella $\operatorname{MotB}_{C}$ is so small that $\operatorname{MotB}(\Delta 51-100)$ cannot reach the PG layer if connected directly to the transmembrane helix by the deletion of residues 51-100. Therefore, a large conformational change would be required for the PGB sites on the top surface of $\mathrm{MotB}_{\mathrm{C}}$ to reach the PG layer. Since a specific interaction between a cytoplasmic loop between TM-2 and TM-3 in MotA and FliG is required for stator assembly around a rotor of the motor, it is possible that the interaction between MotA and FliG may trigger conformational changes in $\operatorname{MotB}_{C}$ that open the proton channel and allow the stator to be anchored to the PG layer (Fig. 9).

Mot $\mathrm{A}$ and MotB are colored blue and orange, respectively. Arg-90 of MotA, which is required for stator assembly into the motor, is shown as a black dot. An interaction between MotA and FliG may trigger conformational changes in $\mathrm{MotB}_{C}$ that open the proton channel and allow the stator to be anchored to the PG layer (Kojima et al., 2009; Morimoto et al., 2010b).

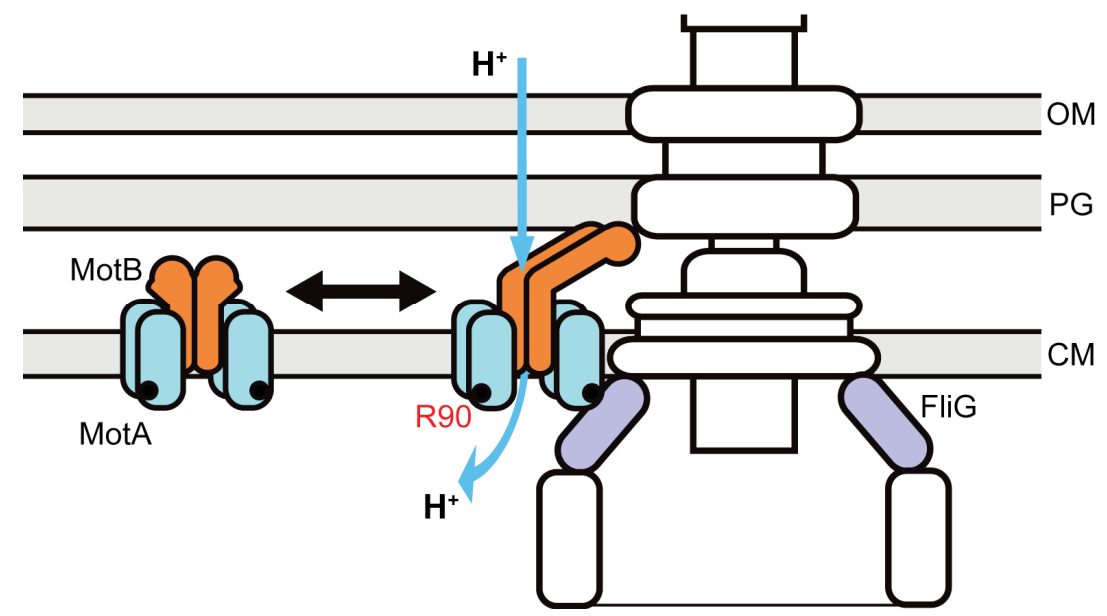

Fig. 9. Model for stator assembly into the motor

\section{Conclusion}

The MotA/B complex, which is composed of four copies of MotA and two copies of MotB, acts as a proton channel to couple proton flow to torque generation. The crystal structure of the Cterminal periplasmic domain of $\operatorname{Mot} B\left(\operatorname{Mot}_{C}\right)$ shows significant structure similarities to other PGB domains such as the C-terminal regions of PAL and RmpM, suggesting that an association of $\mathrm{MotB}_{\mathrm{C}}$ with the PG layer anchors the MotA/B complex to be the stator around the rotor. Interestingly, the MotA/B complex does not always associate with a motor during flagellar motor rotation, suggesting that the association of the MotA/B complex to its target sites on the basal body is highly dynamic. A plug segment in the periplasmic domain of MotB prevents the proton channel from leaking protons into the cytoplasm when the MotA/B complex is not assembled into the motor. An electrostatic interaction between MotA and FliG is required not only for the efficient assembly of the MotA/B complexes around the rotor but also for the proton channel formation to conducts protons coupled with torque generation. 


\section{Acknowledgment}

We acknowledge K. Namba for continuous support and encouragement and N. Kami-ike and Y-S. Che and S. Nakamura for helpful discussions. Y.V.M. is a research fellow of the Japan Society for the Promotion of Science. This work has been supported in part by Grantsin-Aid for Scientific Research from the Ministry of Education, Culture, Sports, Science and Technology of Japan to Y.V.M. and T.M.

\section{References}

Berg, H.C. (2003) The rotary motor of bacterial flagella. Annu Rev Biochem 72: 19-54.

Blair, D.F., \& Berg, H.C. (1988) Restoration of torque in defective flagellar motors. Science 242: 1678-1681.

Blair, D.F., \& Berg, H.C. (1990) The MotA protein of E. coli is a proton-conducting component of the flagellar motor. Cell 60: 439-449.

Blair, D. F. (2003) Flagellar movement driven by proton translocation. FEBS Lett 545: 86-95.

Block, S.M., \& Berg, H.C. (1984) Successive incorporation of force-generating units in the bacterial rotary motor. Nature 309: 470-472.

Braun, T. \& Blair, D.F. (2001) Targeted Disulfide Cross-Linking of the MotB Protein of Escherichia coli: Evidence for Two H+ Channels in the Stator Complex. Biochemistry 40: 13051-13059.

Braun, T., Poulson, S., Gully, J.B., Empey, J.C., Van Way, S., Putnam, A., \& Blair, D.F. (1999) Function of proline residues of MotA in torque generation by the flagellar motor of Escherichia coli. J Bacteriol 181: 3542-3551.

Braun, T.F., Al-Mawasawi, L.Q., Kojima, S. \& Blair, D.F. (2004) Arrangement of core membrane segments in the MotA/MotB protein-channel complex of Escherichia coli. Biochemistry 43: 35-45.

Che, Y-S., Nakamura, S., Kojima S., Kami-ike, N., Namba, K. \& Minamino, T. (2008) Suppressor analysis of the MotB(D33E) mutation to probe the bacterial flagellar motor dynamics coupled with proton translocation. J Bacteriol 190: 6660-6667.

Chen, X. \& Berg, H. C. (2000a) Torque-speed relationship of the flagellar motor of Escherichia coli. Biophys J 78: 1036-1041.

Chen, X. \& Berg, H. C. (2000b) Solvent-isotope and $\mathrm{pH}$ effects on flagellar rotation in Escherichia coli. Biophys J 78: 2280-2284.

De Mot, R. \& Vanderleyden, J. (1994) The C-terminal sequence conservation between OmpA-related outer membrane proteins and MotB suggests a common function in both gram-positive and gram-negative bacteria, possibly in the interaction of these domains with peptidoglycan. Mol Microbiol 12: 333-334.

DeRosier, D. (2006) Bacterial Flagellum: Visualizing the Complete Machine In Situ. Current Biology 16: 928-930.

Fukuoka, H., Wada, T., Kojima, S., Ishijima, A. \& Homma M. (2009) Sodium-dependent dynamic assembly of membrane complexes in sodium-driven flagellar motors. Mol Microbiol 71: 825-835.

Gabel, C.V. \& Berg, H.C. (2003) The speed of the flagellar rotary motor of Escherichia coli varies linearly with protonmotive force. Proc Natl Acad Sci USA 100: 8748-8751.

Hizukuri, Y., Kojima, S. \& Homma, M. (2010) Disulfide cross-linking between the stator and the bearing components in the bacterial flagellar motor. J Biochem 148: 309-318. 
Hosking, E.R., Vogt, C., Bakker, E.P. \& Manson, M.D. (2006) The Escherichia coli MotAB proton channel unplugged. J Mol Biol 364: 921-937.

Kihara, M. \& Macnab, R.M. (1981) Cytoplasmic $\mathrm{pH}$ mediates $\mathrm{pH}$ taxis and weak-acid repellent taxis of bacteria. J Bacteriol 145: 1209-1221.

Kojima, S. \& Blair, D.F. (2001) Conformational change in the stator of the bacterial flagellar motor. Biochemistry 40: 13041-13050.

Kojima, S. \& Blair, D.F. (2004) Solubilization and purification of the MotA/MotB complex of Escherichia coli. Biochemistry 43: 26-34.

Kojima, S., Furukawa, Y., Matsunami, H., Minamino, T. \& Namba, K. (2008) Characterization of the periplasmic domain of MotB and implications for its role in the stator assembly of the bacterial flagellar motor. J Bacteriol 190: 2259-2266.

Kojima, S., Imada, K., Sakuma, M., Sudo, Y., Kojima, C., Minamino, T., Homma, M. \& Namba, K. (2009) Stator assembly and activation mechanism of the flagellar motor by the periplasmic region of MotB. Mol Microbiol 73: 710-718.

Kudryashev, M., Cyrklaff, M., Wallich, R., Baumeister, W. \& Frischknecht, F. (2010) Distinct in situ structures of the Borrelia flagellar motor. J Struct Biol 169:54-61.

Leake, M.C., Chandler, J.H., Wadhams, G.H., Bai, F., Berry, R.M. \& Armitage, J.P. (2006) Stoichiometry and turnover in single, functioning membrane protein complexes. Nature 443: 355-358.

Liu, J., Lin, T., Botkin, D.J., McCrum, E., Winkler, H. \& Norris, S.J. (2009) Intact Flagellar Motor of Borrelia burgdorferi Revealed by Cryo-Electron Tomography: Evidence for Stator Ring Curvature and Rotor/C-Ring Assembly Flexion. J Bacteriol 191: 5026-5036.

Minamino, T., Imae, Y., Oosawa, F., Kobayashi, Y. \& Oosawa, K. (2003) Effect of intracellular $\mathrm{pH}$ on the rotational speed of bacterial flagellar motors. J Bacteriol 185: 1190-1194.

Minamino, T., Imada, K. \& Namba, K. (2008) Molecular motors of the bacterial flagella. Curr Opin Struct Biol 18:693-701.

Morimoto, Y.V., Che, Y-.S., Minamino, T. \& Namba, K. (2010a) Proton-conductivity assay of plugged and unplugged MotA/B proton channel by cytoplasmic pHluorin expressed in Salmonella. FEBS lett 584: 1268-1272.

Morimoto, Y.V., Nakamura, S., Kami-ike, N., Namba, K. \& Minamino, T. (2010b) Charged residues in the cytoplasmic loop of MotA are required for stator assembly into the bacterial flagellar motor. Mol Microbiol 78: 1117-1129

Muramoto, K. \& Macnab, R.M. (1998) Deletion analysis of MotA and MotB, components of the force-generating unit in the flagellar motor of Salmonella. Mol Microbiol 29: 1191-202.

Murphy, G.E., Leadbetter, J.R. \& Jensen, G.J. (2006) In situ structure of the complete Treponema primitia flagellar motor. Nature 442: 1062-1064.

Nakamura, S., Kami-ike, N., Yokota, P.J., Kudo, S., Minamino, T. \& Namba, K. (2009a) Effect of intracellular $\mathrm{pH}$ on the torque-speed relationship of bacterial proton-driven flagellar motor. J Mol Biol 386: 332-338.

Nakamura, S., Morimoto, Y.V., Kami-ike, N., Minamino, T. \& Namba, K. (2009b) Role of a conserved prolyl residue (Pro-173) of MotA in the mechanochemical reaction cycle of the proton-driven flagellar motor of Salmonella. J Mol Biol 393: 300-307.

Nakamura, S., Kami-ike, N., Yokota, J.P., Minamino, T. \& Namba, K. (2010) Evidence for symmetry in the elementary process of bidirectional torque generation by the bacterial flagellar motor. Proc Natl Acad Sci USA 107: 17616-17620. 
Reid, S.W., Leake, M.C., Chandler, J.H., Lo, C.J., Armitage, J.P. \& Berry, R.M. (2006) The maximum number of torque-generating units in the flagellar motor of Escherichia coli is at least 11. Proc Natl Acad Sci USA 103: 8066-8071.

Ryu, W.S., Berry, R.M. \& Berg, H.C. (2000) Torque-generating units of the flagellar motor of Escherichia coli have a high duty ratio. Nature 403: 444-447.

Sharp, L.L. Zhou, J. \& Blair, D.F. (1995) Tryptophan-scanning mutagenesis of MotB, an integral membrane protein essential for flagellar rotation in Escherichia coli, Biochemistry 34, 9166-9171.

Sowa, Y., Rowe, A.D., Leake, M.C., Yakushi, T., Homma, M., Ishijima, A. \& Berry, R.M. (2005) Direct observation of steps in rotation of the bacterial flagellar motor. Nature 437: 916-919.

Stolz, B. \& Berg, H.C. (1991) Evidence for interactions between MotA and MotB, torquegenerating elements of the flagellar motor of Escherichia coli. J Bacteriol 173: 7033-7037.

Thomas, D., Morgan, D.G. \& DeRosier, D.J. (2001) Structures of bacterial flagellar motors from two FliF-FliG gene fusion mutants. J Bacteriol 183: 6404-6412.

Thomas, D.R., Francis, N.R., Chen, X. \& DeRosier, D.J. (2006) The three-dimensional structure of the flagellar rotor from a clockwise-locked mutant of Salmonella enterica serovar Typhimurium. J Bacteriol 188: 7039-7048.

Togashi, F., Yamaguchi, S., Kihara M., Aizawa, S.-I. \& Macnab, R.M. (1997) An extreme clockwise switch bias mutation in fliG of Salmonella typhimurium and its suppression by slow-motile mutations in motA and motB. J Bacteriol 179: 2994-3003.

Yuan, J., \& Berg, H.C. (2008) Resurrection of the flagellar rotary motor near zero load. Proc Natl Acad Sci USA 105: 1182-1185.

Zhou, J. \& Blair, D.F. (1997) Residues of the cytoplasmic domain of MotA essential for torque generation in the bacterial flagellar motor. J Mol Biol 273: 428-439.

Zhou, J., Lloyd, S. A. \& Blair, D.F. (1998a) Electrostatic interactions between rotor and stator in the bacterial flagellar motor. Proc Natl Acad Sci USA 95: 6436-6441.

Zhou, J., Sharp, L.L., Tang, H.L., Lloyd, S.A. \& Blair, D.F. (1998b) Function of protonatable residues in the flagellar motor of Escherichia coli: a critical role for Asp32 of MotB. J Bacteriol 180: 2729-2735. 
(C) 2012 The Author(s). Licensee IntechOpen. This is an open access article distributed under the terms of the Creative Commons Attribution 3.0 License, which permits unrestricted use, distribution, and reproduction in any medium, provided the original work is properly cited. 